DOI: $10.1002 /(($ please add manuscript number))

Article type: Communication

3

4

5

6

7

\title{
Click-Chemistry Immobilized 3D-infused Microarrays in Nanoporous Polymer
} Substrates

Michael Hirtz, * Wenqian Feng, Harald Fuchs, and Pavel A. Levkin*

Dr. M. Hirtz, Prof. H. Fuchs

Institute of Nanotechnology (INT) and Karlsruhe Nano Micro Facility (KNMF), Karlsruhe Institute of Technology (KIT), Germany

E-mail: michael.hirtz@kit.edu

W. Feng, Dr. P. A. Levkin

Institute of Toxicology and Genetics (ITG), Karlsruhe Institute of Technology (KIT), Germany

E-mail: pavel.levkin@kit.edu

Prof. H. Fuchs

Physical Institute and Center for Nanotechnology (CeNTech), University of Münster, Germany

Dr. P. A. Levkin

Applied Physical Chemistry, Heidelberg University, Germany

Keywords: 3D microarrays, click-chemistry, microspotting, CuAAC, polymer films 
1 Microarrays are widely used in many fields of biological and biomedical research as well as

2 in medical diagnostics. ${ }^{[1-6]}$ For reliable use, the microarrays should be stable, homogeneous

3 and easy to read out, while at the same time, miniaturizing is desired for reduced use of

4 consumables and sample. Recently, we have shown the advantageous properties of

5 nanoporous HEMA-EDMA polymer for the generation of miniaturized microarrays in

6 comparison to conventional spotting substrates. ${ }^{[7]}$ The microarrays fabricated on the

7 nanoporous HEMA-EDMA polymer could feature smaller spot sizes (at least one order of

8 magnitude less than in typical ink jet printing) while retaining sharper feature edges when

9 compared to other commonly used substrates for spotting as paper, nitrocellulose and nylon membranes. This higher pattern fidelity allows for the reduction of spot size, while still

11 obtaining well-defined features, thus facilitating readout of the arrays and saving materials.

12 However, a substrate with similar properties but functionality for covalent binding of spotted 13 substances would be of great importance to many applications in spotting and sensing, 14 nanostructured surfaces being e.g. used for enhanced capture and detection of bacteria. ${ }^{[8]}$ 15 Alkyne modification of glass substrates ${ }^{[9]}$ allowed for the formation of covalently bound 16 microarrays by spotting with molecules functionalized with the azide group. ${ }^{[10]}$ To combine 17 the favorable properties of the nanoporous polymeric substrates (average pore size $150 \mathrm{~nm})^{[7]}$ with the covalent binding demonstrated on the glass substrates, we here present an alkyne modification of the porous polymer (alkyne-HEMA-EMDA) allowing for click-chemistry coupling of azides via copper(I)-catalyzed azide-alkyne cycloaddition (CuAAC). ${ }^{[1-13]}$ This 21 prominent click reaction found many applications, e.g. in the generation of nanosized 22 molecular junctions. ${ }^{[14]}$ The polymer retains its desirable spotting properties and covalently immobilized high quality microarrays can be generated. The three dimensional impregnation

24 of the polymer with a fluorescently labeled azide is quantified by confocal microscopy and leads to considerably higher spot fluorescence intensities compared to strictly 2-dimensional surfaces as modified glass. Furthermore, the microarrays can be multiplexed and used for 
1 binding of proteins, as is demonstrated by selective binding of streptavidin and specific

2 antibody on an allergen array.

3 Figure 1a shows the schematic spotting setup as used in the present work. A quill-like

4 microchannel cantilevers, called surface patterning tools (SPTs) ${ }^{[15]}$ is brought into contact with

5 the alkyne-HEMA-EMDA surface for defined time lengths allowing the imbibition of

6 spotting solution into the polymer. By high precision scanning in $\mathrm{x}$ - and $\mathrm{y}$ - direction via the

7 stage of a dip-pen nanolithography (DPN) platform (NLP 2000, NanoInk, USA), the spot

8 features of the microarray are generated one after the other. The spotting solution is based on

9 water with admixing of glycerol to prevent drying out. It contains an azide-modified molecule for immobilization, sodium ascorbate and copper sulfate to catalyze the CuAAC reaction. The

11 chemical structure of the used azides is given in Figure $1 \mathrm{~b}$ for tetramethylrhodamine

12 (TAMRA) azide and Figure 1c for biotin azide, respectively.

13 A typical outcome of a spotting with TAMRA azide is shown in Figure 2a. This pattern of 14100 spots arranged in a square with a pitch of $50 \mu \mathrm{m}$ (yielding an array area of $500 \times 500 \mu \mathrm{m}^{2}$ ) was written with a dwell time of $0.5 \mathrm{~s}$ by a single quill-like pen in about one minute.

Generally, the spotting procedure can be parallelized by introduction of pen arrays, ${ }^{[16]}$ yielding even higher throughput with larger areas and additional possibility of intrinsic multiplexing. In our present setup, we realized multiplexing by subsequent writing with tip changing for the different inks, which could principally automated as alternative route to multiplexed arrays. Due to the CuAAC mediated covalent immobilization of desired molecules the pattern

21 becomes highly stable in solution (see Supporting Information, Figure S1 and Figure S2). 22 The average radius of a dot feature in such arrays is $(9.9 \pm 0.5) \mu \mathrm{m}$, a histogram of the radius 23 distribution is given in Figure $2 \mathrm{f}$. To demonstrate the three-dimensional structure of the 24 generated patterns due to imbibition of ink into the three dimensional porous polymer layer, we employed confocal microscopy. A 3D representation of a typical array as obtained from 
1 confocal microscopy is shown in Figure $2 \mathrm{~b}$ with a cross-section through one row of dots

2 shown in Figure 2c. The outlines of the dot features as obtained by a thresholding of

3 fluorescent intensity are added to the section and from this the hemispherical shape of the

4 deposited dot features within the polymer film becomes obvious. To characterize the ink

5 transport process during lithography, dot patterns with varying dwell times were spotted

6 (Figure 2e). The radius of the dot features increases from about $8 \mu \mathrm{m}$ up to $14 \mu \mathrm{m}$ with

7 increasing dwell time from $0.1 \mathrm{~s}$ to $2.0 \mathrm{~s}$ (Figure $2 \mathrm{~g}$ ). For imbibition of a fluid into a porous

8 substrate, theory predicts a dependence on time proportional to $t^{1 / 3} \cdot{ }^{[17]}$ The spot radius vs.

9 dwell time dependence in our system follows this prediction, deviating only for smaller dwell

10 times especially in the case of $0.1 \mathrm{~s}$ (smallest dwell time possible in our setup). This could be

11 an artifact introduced by a combination of the DPN system used contacting the surface some

12 time longer then intended for this small dwell times near the system's limit and the deviation

13 of our SPT (which has an opening at the apex of about $1 \mu \mathrm{m}$ ) from a point source as assumed

14 in the theoretical description. Figure $2 \mathrm{~d}$ contains a section through one row of the dot array in

15 Figure 2e and shows that also the functionalized volume within the polymer film is dependent

16 on dwell time as expected. With the radius $r$ being proportional to $t^{1 / 3}$ it is expected that the

17 volume $V$ of functionalized polymer (being a half sphere) should be direct proportional to $t$.

18 Plotting of the dot feature volume against dwell time (Figure $2 \mathrm{~h}$ ) reveals that our system

19 exhibits a linear dependence on dwell time. Likewise to the radius, even at short dwell time

20 the deposited volume does not reach zero, again reflecting a minimum volume of ink transfer

21 just by touching the surface with a SPT. The volume character of the microarray features

22 leads to a strong increase in fluorescence intensity compared to the strictly 2D nature of the

23 features on glass slides. In the TAMRA-azide spots a 20 -fold increase in signal intensity

24 could be observed (Figure S3). 
1 To demonstrate the capability of the approach for multiplexing and the stability of the

2 microarrays for analyte binding from solution, we created bi-color patterns with alternating

3 TAMRA-azide and biotin-azide spots. This was done by writing TAMRA-azide spots as

4 described above, but leaving each second spot free and then subsequently filling these empty

5 spaces with biotin-azide spots after replacing of the SPT. Biotin can serve as a strong binding

6 motive for streptavidin, ${ }^{[18]}$ effectively immobilizing the protein onto the biotin-azide

7 functionalized spots in the array (Figure 3a). Since the biotin-azide is not fluorescent, only

8 the TAMRA-azide spots are visible in fluorescent images of the array prior to fluorescently

9 labeled streptavidin binding (Figure 3b). After incubation of the microarray with a solution

10 containing fluorescein conjugated streptavidin, the prior invisible biotin-azide spots light up

11 in the green channel of the fluorescent imaging indicating successful and selective binding of

12 the protein.

13 To further elucidate the prospects of the polymer coating based microarrays for sensing and

14 binding of biological relevant proteins, we compared the binding of antibodies to antigen15 arrays on glass slides and alkyne-HEMA-EDMA polymer substrates (Figure 4). Glass was 16 chosen as comparison for its broad use in similar assays, and to clearly contrast the 3D 17 infused arrays to purely 2D ones (no diffusion in or swelling of substrate). For this 18 experiment, microarrays of dinitrophenol (DNP)-azide were patterned on alkyne modified 19 glass and alkyne-HEMA-EDMA substrates as described above. DNP can act - among other $20 \mathrm{uses}^{[19]}$ - as a model allergen for the activation of mast cells, ${ }^{[20-23]}$ here we use it as target for 21 specific antibody binding. ${ }^{[24,25]}$ After spotting and washing of the samples, solutions with 22 different concentrations of fluorescently labelled anti-DNP antibodies (starting from $10 \mu \mathrm{g} / \mathrm{ml}$ 23 in the highest concentration and then diluted 1:1 by volume with PBS for each following 24 dilution step). Figure 4a shows a typical outcome of such antibody binding to a DNP 25 microarray on an alkyne-HEMA-EDMA sample, showing clearly preferential binding of the 
1 antibody to the DNP features of the array. Quantifying the resulting fluorescence signals for

2 the series of antibody dilutions on alkynized HEMA-EDMA and comparison with the analog

3 experiment on glass substrate samples further validates the discussed feature volume effect

4 (Figure 4b): while for low antibody concentrations (0.125 and 0.250 relative antibody

5 concentration) both substrate systems behave similar, the signal on the glass substrate levels

6 off, while on alkyne-HEMA-EDMA substantially higher intensities are found for increasing

7 antibody concentration ( 0.500 and 1.000 relative concentration). This behavior indicates, that

8 the accessible binding sites (i.e. DNP moieties) on the glass slide are already completely

9 saturated on the glass slide at the 0.250 relative antibody concentration. Therefore, further

10 increase in antibody concentration cannot result in an increased fluorescence signal. On the

11 alkyne-HEMA-EDMA substrate, additional binding sites are available in the bulk volume of

12 the feature, hence enabling further increase in binding and fluorescence intensity on higher

13 relative concentrations.

14 In conclusion, we present a novel platform for the immobilization of azide functionalized 15 compounds and subsequently protein binding. The use of quill-like pens enables the delivery 16 of higher ink volumes than e.g. microcontact printing or similar methods, therefore allowing 17 infiltration into the porous substrate. This gives increased intensity from fluorescent 18 compounds since the whole functionalized volume can contribute to the signal. Additionally, 19 the method allows for multiplexed arrays with different compounds within one array. The 20 micropatterns show high stability under immersion in solvents and can be used for selective 21 binding of proteins as e.g. streptavidin or antibodies. The volume nature of the microarray 22 features on alkyne-HEMA-EDMA substrate allows for additional binding sites when using 23 the arrays as protein targets, resulting in a higher dynamic range of quantifiable 24 concentrations when compared to 2D microarrays on non-porous substrates. While the porous 25 nature of the functionalized volume limits the access by liquid exchange and diffusion speed 
1 to this additional sensitive area, the higher dynamic range gained enables testing of a much

2 wider range of target concentrations, allowing easier application of such assays. As the overall

3 size of pores is in the range of $150 \mathrm{~nm}$, this sets an upper limit of target size, though typical

4 protein targets will be not inflicted, as they are much smaller. Overall, these properties make

5 the presented porous polymer film an attractive substrate for microarraying e.g. for bio-

6 medical or environmental testing.

\section{Experimental Section}

8 Substrate preparation: The fabrication of alkyne-HEMA-EDMA polymer film was described

9 in our previous work. ${ }^{[2]}$ Briefly, a $12.5 \mu \mathrm{m}$ thin, hydrophilic porous polymer film was firstly prepared on a glass substrate using photoinitiated copolymerization of 2-hydroxyethyl

11 methacrylate and ethylene dimethacrylate in the presence of porogens (non-alkynized HEMA-

12 EDMA) ${ }^{[27]}$ This procedure leads to the formation of a thin film of a highly cross-linked and 13 porous (due to the presence of porogens) polymethacrylate layer. The porous structure is 14 permeable network of interconnected polymer nanoglobules with pores of around $150 \mathrm{~nm}$.

15 The polymer surface was esterified by immersing into a dichloromethane solution containing 16 4-pentynoic acid $(22.8 \mathrm{mM})$, coupling reagent $N, N^{\prime}$-diisopropylcarbodiimide $(22.8 \mathrm{mM})$, and 17 catalyst 4-(dimethylamino)pyridine $(9.2 \mathrm{mM})$ and stirring at RT for $4 \mathrm{~h}$. A porous HEMA18 EDMA layer functionalized with alkyne groups (alkyne-HEMA-EDMA) was produced.

Spotting Setup: The spotting procedures were done on a NLP 2000 system (NanoInk, USA) equipped with SPT pens (SPT-S-C10S, Bioforce Nanosciences). Prior to use, the SPTs were

21 freshly plasma cleaned by oxygen plasma (10 sccm O2, $100 \mathrm{mTorr}, 30 \mathrm{~W}$ for $2 \mathrm{~min})$ and used immediately. The SPT was mounted onto the tip holder by double-sided sticky tape, and the pen reservoir was filled with $1 \mu \mathrm{L}$ of azide solution. All spotting procedures were 24 implemented at a relative humidity of $60 \%$ and with the sample stage tilted by $8^{\circ}$ with respect to the SPT tip to minimize the chance of contact between the ink reservoir and the sample 
1 surface. For all patterns, except for the pattern used for spot size versus dwell time analysis, a

2 dwell time of $0.5 \mathrm{~s}$ was used.

3 Streptavidin binding: After the spotting process, the samples were washed extensively with DI

4 water $(18.2 \mathrm{M} \Omega \mathrm{cm})$ to remove excess ink. Prior to adding the streptavidin solution, samples

5 were blocked with $5 \%$ bovine serum albumin $(\mathrm{BSA})^{[28]}$ (Sigma-Aldrich) in phosphate

6 buffered saline (PBS) (Gibco) for 10 minutes. Subsequently, the samples were washed 3 times

7 by pipetting on and off $100 \mu$ of PBS ( 3 times each) and then incubated for 10 minutes with a

8 solution of $5 \mu \mathrm{g} / \mathrm{ml}$ of streptavidin fluorescein conjugate (Calbiochem/Merck). After repeating

9 the washing steps, samples were imaged with fluorescence microscopy.

Antibody Binding: Microarrays of DNP-azide were generated on alkyne-HEMA-EMDA and

11 glass substrates (glass substrates alkyne modification was performed as described in the

12 literature $)^{[9,10]}$ as described above and washed with DI water $(18.2 \mathrm{M} \Omega \mathrm{cm})$ to remove excess

13 ink. Then, the samples were blocked with 5\% BSA (Sigma-Aldrich) in PBS (Gibco) for 10

14 minutes. Subsequently, the samples were washed 3 times by pipetting on and off $100 \mu l$ of

15 PBS (3 times each) and then incubated with different concentrations of mouse anti-DNP

16 antibody previously fluorescently labelled by a Mix-n-Stain CF 488A antibody labelling kit

17 (both Sigma-Aldrich, Germany) for 60 minutes. The highest concentration of antibody was

$1810 \mu \mathrm{g} / \mathrm{ml}$ and more and more diluted solutions were created by admixing the previous solution

$191: 1$ by volume with PBS, resulting in relative concentrations of $0.500,0.250$, and 0.125 ,

20 respectively, compared to the highest concentration. After incubation, the samples were

21 washed 3 times again and then imaged by fluorescence microscopy.

22 Imaging setup: Fluorescent microscopic images were obtained on an Eclipse 80i upright

23 fluorescence microscope (Nikon) equipped with an Intensilight (Nikon) for illumination and a 
1 CoolSNAP HQ2 camera (Photometrics). The confocal images were recorded using a Leica

2 TCS SP5 confocal microscope.

3 Image analysis: The fluorescent microscopy images used for the determination of size

4 distribution and influence of dwell time were analysed with ImageJ. ${ }^{[29,30]}$ After converting the

5 images to black and white by a threshold filter, the spot sizes were obtained by particle

6 analyses. Particles smaller than 20 pixels were excluded from analysis to exclude noise-

7 induced artefacts. To determine the functionalized polymer volume from the confocal images,

8 this procedure was applied to each layer of the image stack, the resulting areas multiplied with

9 the thickness of one layer and associated volume slices for each point were summed up.

\section{Supporting Information}

12 Supporting Information is available from the Wiley Online Library or from the author.

[1] V. Romanov, S. N. Davidoff, A. R. Miles, D. W. Grainger, B. K. Gale, B. D. Brooks,

\section{Acknowledgements}

This work was carried out with the support of the Karlsruhe Nano Micro Facility (KNMF, www.kmf.kit.edu), a Helmholtz Research Infrastructure at Karlsruhe Institute of Technology (KIT, www.kit.edu). The research is supported by the ERC Starting Grant (ID: 337077DropCellArray) and the Helmholtz Association's Initiative and Networking Fund (grant VHNG-621).

Received: ((will be filled in by the editorial staff))

Revised: ((will be filled in by the editorial staff)) Published online: ((will be filled in by the editorial staff))

[2] M. B. Miller, Y. W. Tang, Clin. Microbiol. Rev. 2009, 22, 611-633.

[3] J. M. Lucas, Allergol. Immunopathol. (Madr). 2010, 38, 217-23.

[4] J. M. Lucas, Allergol. Immunopathol. (Madr). 2010, 38, 153-61. Academic Press, 2014. 
1 [6] H. Chandra, P. J. Reddy, S. Srivastava, Expert Rev. Proteomics 2011, 8, 61-79.

[7] M. Hirtz, M. Lyon, W. Feng, A. E. Holmes, H. Fuchs, P. A. Levkin, Beilstein J. Nanotechnol. 2013, 4, 377-384.

[8] Y.-Q. Li, B. Zhu, Y. Li, W. R. Leow, R. Goh, B. Ma, E. Fong, M. Tang, X. Chen, Angew. Chemie Int. Ed. 2014, 53, 5837-5841.

[9] S. Oberhansl, M. Hirtz, A. Lagunas, R. Eritja, E. Martinez, H. Fuchs, J. Samitier, Small 2012, $8,541-545$.

[10] M. Hirtz, A. M. Greiner, T. Landmann, M. Bastmeyer, H. Fuchs, Adv. Mater. Interfaces 2014, 1, 1300129.

[11] V. V Rostovtsev, L. G. Green, V. V Fokin, K. B. Sharpless, Angew. Chemie Int. Ed. 2002, 41, 2596-9.

[12] C. W. Tornøe, C. Christensen, M. Meldal, J. Org. Chem. 2002, 67, 3057-64.

[13] L. Liang, D. Astruc, Coord. Chem. Rev. 2011, 255, 2933-2945.

[14] X. Chen, A. B. Braunschweig, M. J. Wiester, S. Yeganeh, M. A. Ratner, C. A. Mirkin, Angew. Chemie Int. Ed. 2009, 48, 5178-5181.

[15] J. Xu, M. Lynch, J. L. Huff, C. Mosher, S. Vengasandra, G. Ding, E. Henderson, Biomed. Microdevices 2004, 6, 117-23.

[16] J. Xu, M. Lynch, S. Nettikadan, C. Mosher, S. Vegasandra, E. Henderson, Sensors Actuators B Chem. 2006, 113, 1034-1041.

[17] J. Xiao, H. A. Stone, D. Attinger, Langmuir 2012, 28, 4208-12.

[18] N. M. Green, Adv. Protein Chem. 1975, 29, 85-133.

[19] H. C. H. Lee, C. Y. Law, M. L. Chen, Y. H. Lam, A. Y. W. Chan, T. W. L. Mak, J. Chinese Med. Assoc. 2014, 77, 443-445.

[20] R. N. Orth, M. Wu, D. A. Holowka, H. G. Craighead, B. A. Baird, Langmuir 2003, 19, $1599-1605$.

[21] S. Sekula-Neuner, J. Maier, E. Oppong, A. C. B. Cato, M. Hirtz, H. Fuchs, Small 2012, $8,585-591$.

[22] E. Oppong, P. N. Hedde, S. Sekula-Neuner, L. Yang, F. Brinkmann, R. M. Dörlich, M. Hirtz, H. Fuchs, G. U. Nienhaus, A. C. B. Cato, Small 2014, 10, 1991-1998.

[23] D. Jiang, J. Ji, L. An, X. Sun, Y. Zhang, G. Zhang, L. Tang, Biosens. Bioelectron. 2013, 50, 150-156. 
[24] C. A. J. Janeway, P. Travers, M. Walport, M. J. Shlomchik, Immunobiology, Garland Science, New York, 2001.

[25] G. W. Litman, J. P. Rast, M. J. Shamblott, R. N. Haire, M. Hulst, W. Roess, R. T. Litman, K. R. Hinds-Frey, A. Zilch, C. T. Amemiya, Mol. Biol. Evol. 1993, 10, 60-72.

[26] W. Feng, L. Li, E. Ueda, J. Li, S. Heißler, A. Welle, O. Trapp, P. a. Levkin, $A d v$. Mater. Interfaces 2014, 1, 1400269.

[27] F. L. Geyer, E. Ueda, U. Liebel, N. Grau, P. A. Levkin, Angew. Chemie Int. Ed. 2011, $50,8424-7$.

[28] H. Schønheyder, P. Andersen, J. Immunol. Methods 1984, 72, 251-9.

[29] M. D. Abràmoff, P. J. Magalhães, S. J. Ram, Biophotonics Int. 2004, 11, 36-42.

[30] C. A. Schneider, W. S. Rasband, K. W. Eliceiri, Nat. Methods 2012, 9, 671-675.

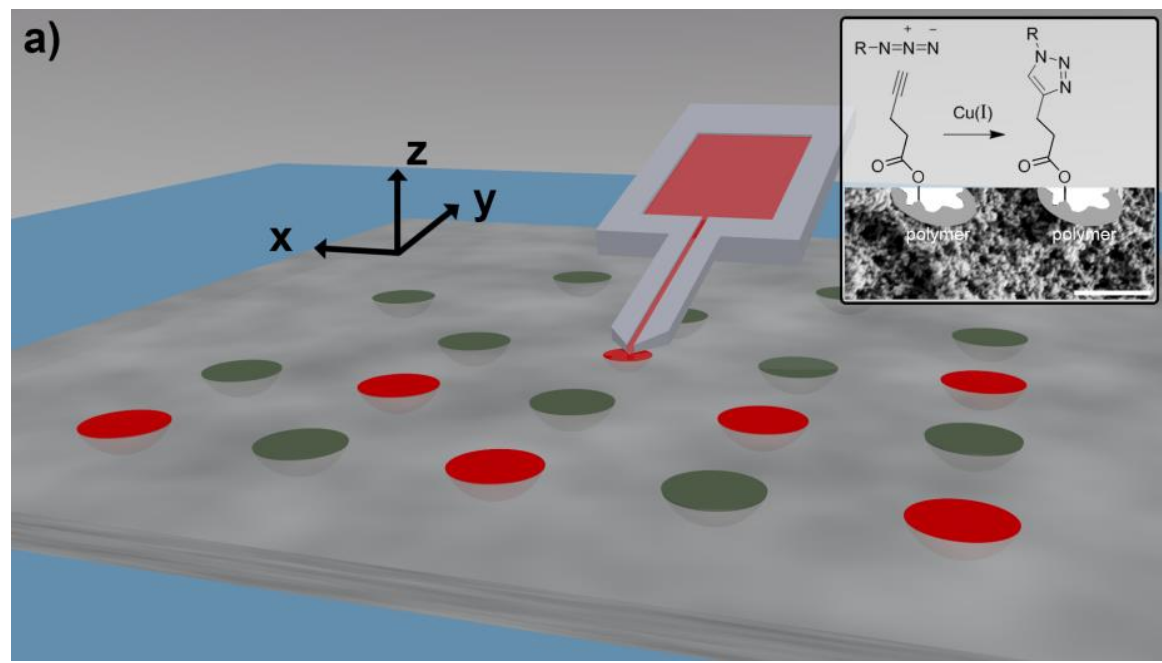

b)

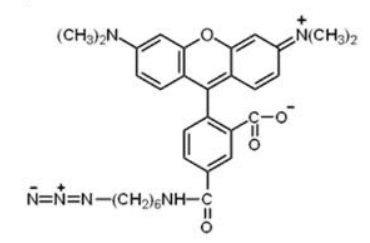

c)

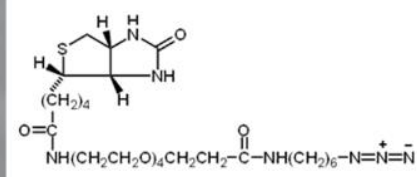

Figure 1. (a) Schematic view of the patterning process on the porous polymers. The inset shows the CuAAC reaction taking place on the alkyne-HEMA-EMDA substrate. Structures of the azides that were immobilized are shown in (b) for TAMRA azide and (c) for biotin azide. 

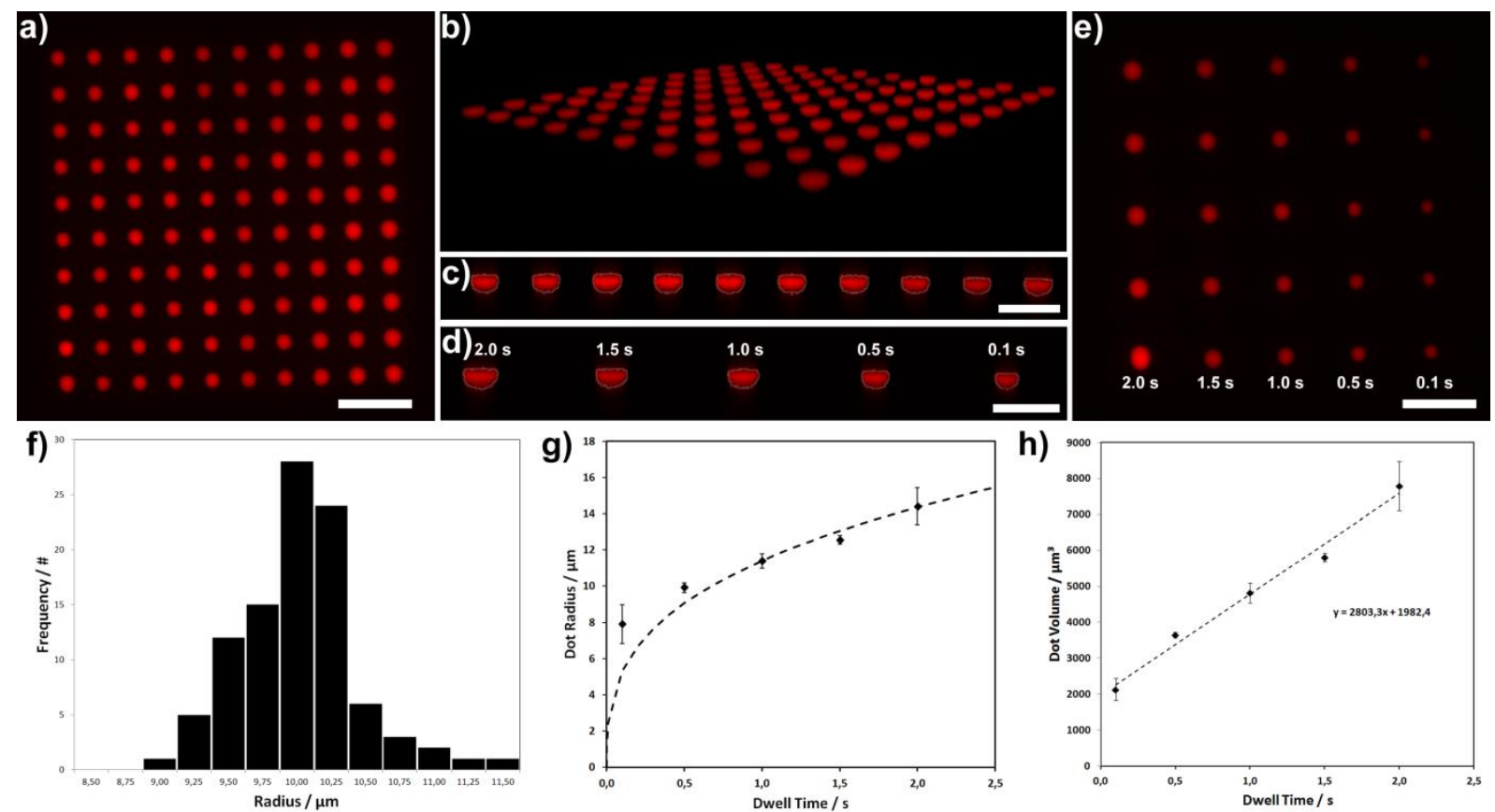

Figure 2. (a) Fluorescent microscopy image of a pattern TAMRA azide dot array. Scale bar equals $100 \mu \mathrm{m}$. (b) $3 \mathrm{D}$ image of a $10 \times 10$ dot array obtained with confocal microscopy to determine the volume distribution of the TAMRA azide within the polymer film. (c) Section through a line of dots from (b). Scale bar equals $50 \mu \mathrm{m}$, dot volume is outlined. (d) Section through a line of dots from (e) written with different dwell times. Scale bar equals $50 \mu \mathrm{m}$, dot volume is outlined. (e) $5 \times 5$ dot array with columns written with different dwell times. Scale bar equals $100 \mu \mathrm{m}$. (f) Histogram of the radius distribution of 100 dot features written at a dwell time of $0.5 \mathrm{~s}$. (g) Dependence of the dot radius on dwell time. The dashed line follow $\mathrm{r}$ $=11.4 \mathrm{t}^{1 / 3}$. (h) Dependence of dot volume on dwell time. The dashed line follows $r=2803.3 \mathrm{t}$ +1982.4 . 

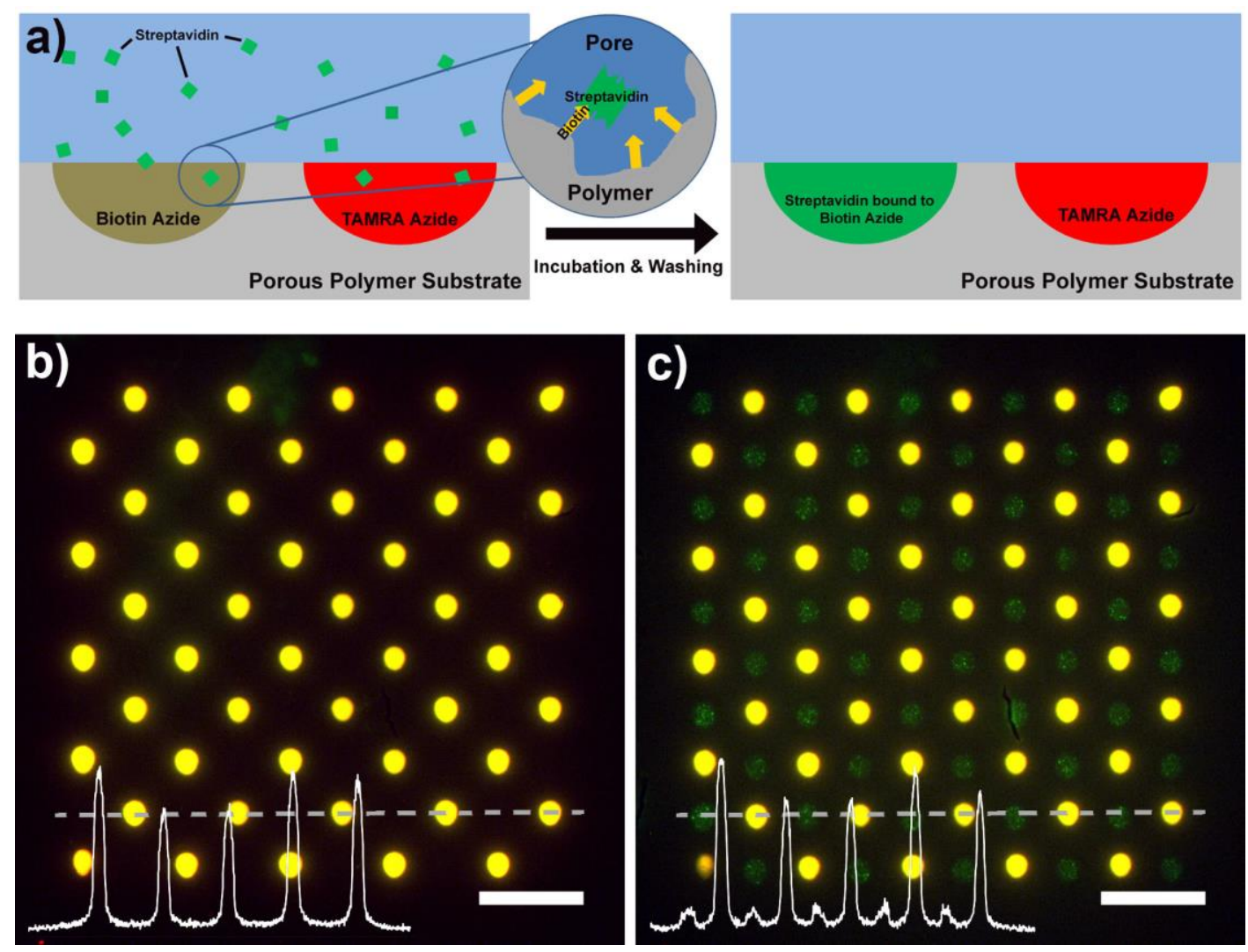

Figure 3. (a) Scheme of binding of streptavidin to multiplexed biotin- / TAMRA-azide microarrays. (b) Fluorescent microscopy image (green and red channel combined) of a multiplexed biotin- / TAMRA-azide microarray after washing. Only the TAMRA containing spots are visible. (c) Same microarray after incubation with fluorescein conjugated streptavidin. Now the previously invisible biotin-azide spots light up in the green channel. All scale bars equal $100 \mu \mathrm{m}$. The insets in the lower left of (b) and (c) show fluorescence intensity along the dashed lines.
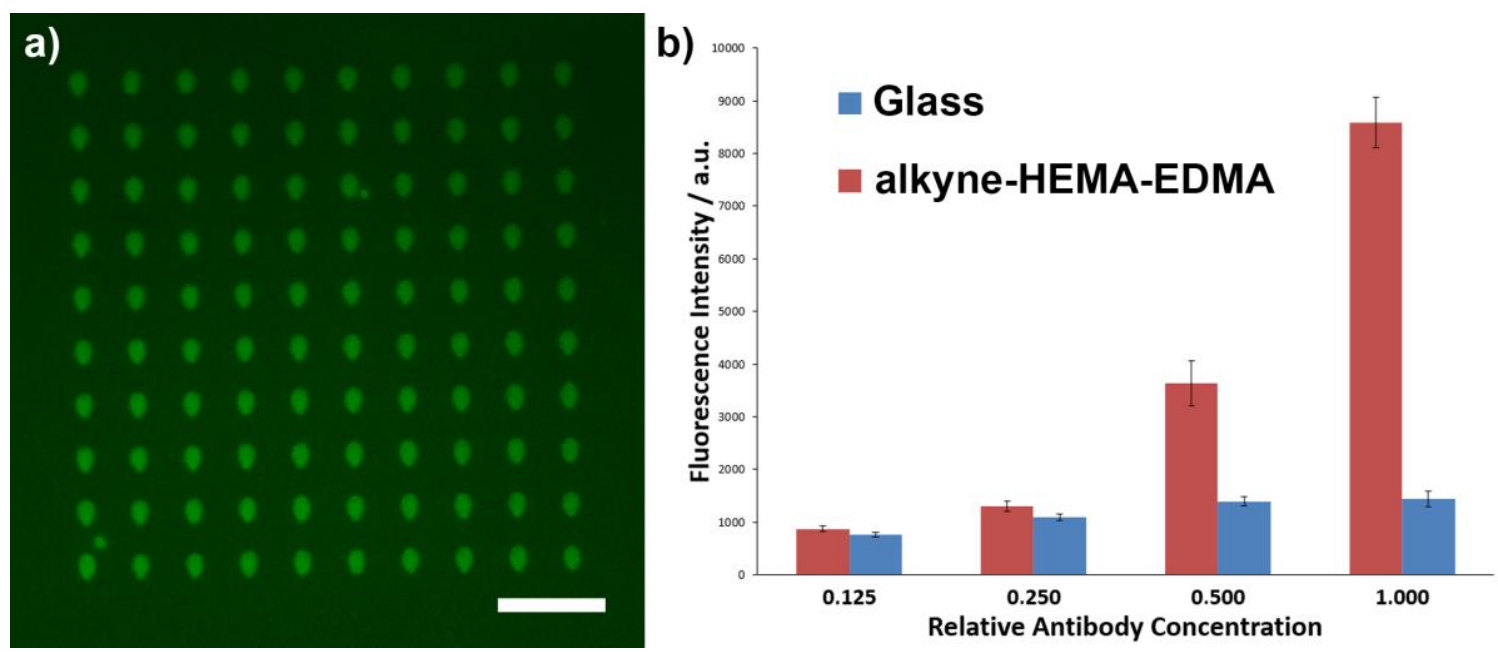

Figure 4. (a) Fluorescence microscopy image of a DNP-azide microarray on an alkynized HEMA-EDMA substrate after incubation with fluorescently labelled anti-DNP-antibody. Scale bar equals $100 \mu \mathrm{m}$. (b) Comparison of fluorescent intensity of the microarrays features on glass and alkyne-HEMA-EDMA substrates after incubation with different concentrations of antibodies. 
3-Dimensional microarrays are created in a nanoporous polymer film. The microarrays are stabilized by covalent attachment over a click-chemistry approach and exhibit an enhanced dynamic range for the detection of antibody binding due to a higher amount of binding sites in the nanoporous film compared to flat substrates with conventional 2-dimensional surface arrays.

\section{D Microarrays}

M. Hirtz*, W. Feng, H. Fuchs, and P. A. Levkin*

10

Click-Chemistry Immobilized 3D-infused Microarrays in Nanoporous Polymer Substrates

13
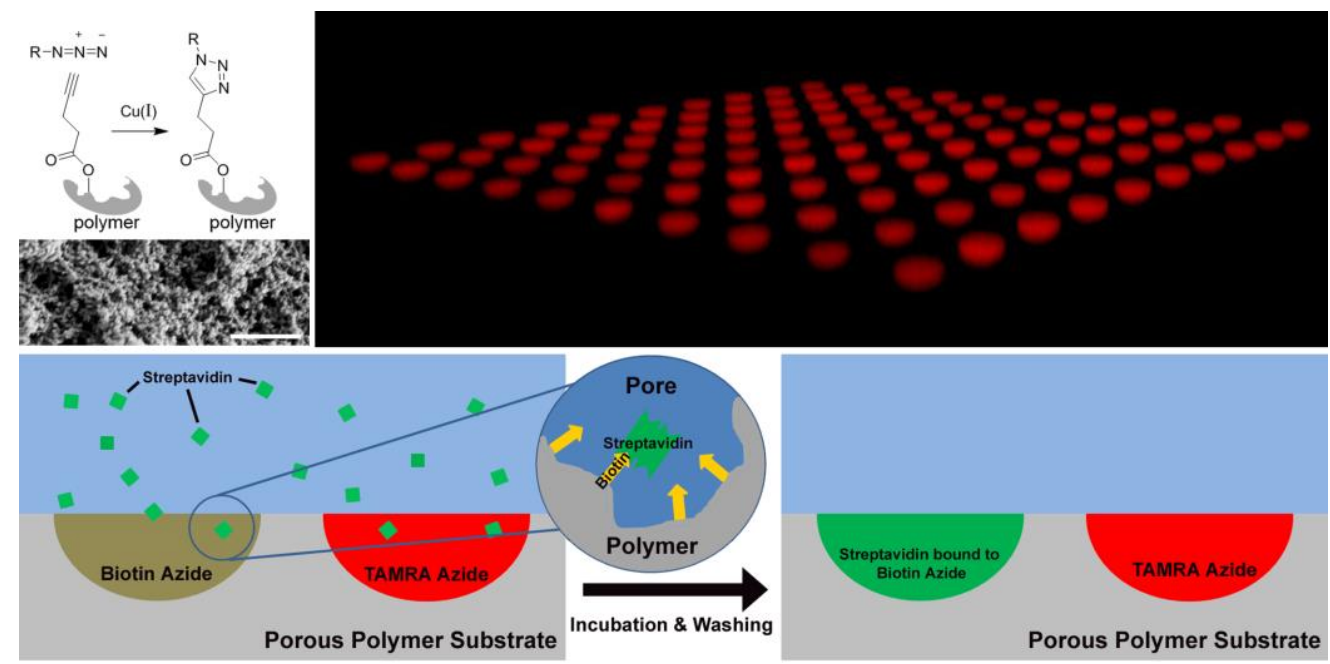

15

16 